\title{
ESTUDO COMPARATIVO DAS SEQÜÊNCIAS RÁPIDAS PONDERADAS EM T2, UTILIZANDO-SE SINCRONIZAC̣ÃO RESPIRATÓRIA, APNÉIA, SUPRESSÃO DE GORDURA, BOBINA DE CORPO E BOBINA DE SINERGIA PARA A AVALIAC̣ÃO DO FÍGADO PELA RESSONÂNCIA MAGNÉTICA*
}

\author{
Cristiane L. Abbehusen ${ }^{1}$, Giuseppe D'Ippolito ${ }^{2}$, Glaucia A.S. Palácio ${ }^{1}$, Jacob Szejnfeld ${ }^{3}$
}

Resumo OBJETIVO: Comparar, qualitativa e quantitativamente, as imagens de ressonância magnética do fígado, ponderadas em T2, utilizando-se seqüências rápidas, diferenciadas pela técnica de controle respiratório, pela utilização de supressão de gordura e pelo tipo de bobina de radiofreqüência. MATERIAIS E MÉTODOS: Estudo prospectivo em 71 pacientes consecutivos, sendo realizadas seis seqüências para comparação: 1) supressão de gordura com sincronização respiratória e bobina de corpo; 2) supressão de gordura em apnéia e bobina de corpo; 3) sem supressão de gordura com sincronização respiratória e bobina de corpo; 4) sem supressão de gordura em apnéia e bobina de corpo; 5) com supressão de gordura com sincronização respiratória e bobina de sinergia; 6) com supressão de gordura em apnéia e bobina de sinergia. A avaliação qualitativa foi baseada em três critérios: detecção de determinadas estruturas anatômicas do fígado, definição dos contornos hepáticos, e presença de artefatos de respiração. A análise quantitativa foi obtida através da relação das intensidades de sinal do fígado e do ruído de fundo. RESULTADOS: 0 valor médio dos índices globais de qualidade de imagem para cada uma das seis seqüências supracitadas foi de 7,8, 4,6, 7,9, 5,2, 6,7 e 4,6, respectivamente. As seqüências obtidas com sincronização respiratória apresentaram melhor qualidade de imagem e relação sinal/ruído superiores às seqüências com apnéia ( $p<0,001)$. As seqüências realizadas com e sem supressão de gordura apresentaram qualidade de imagem e relação sinal/ruído semelhantes $(p>0,05)$. As seqüências obtidas com bobina de sinergia apresentaram qualidade de imagem semelhante $(p>0,05)$ e relação sinal/ruído inferior àquelas com bobina de corpo ( $p<0,001)$. CONCLUSÃO: Associando-se as análises qualitativa e quantitativa das imagens, as melhores seqüências foram aquelas obtidas com sincronização respiratória e bobina de corpo, utilizando-se ou não supressão de gordura.

Unitermos: Imagem por ressonância magnética; Fígado; Técnicas de diagnóstico do sistema digestório; Técnicas de imagem.

Abstract Comparative study of fast T2-weighted images using respiratory triggered, breath-hold, fat suppression and phased array multicoil for liver evaluation by magnetic resonance imaging.

OBJECTIVE: To compare both qualitatively and quantitatively six T2-weigthed turbo spin-echo sequences varying the respiratory compensation technique, associating or not fat tissue suppression and using different types of coils. MATERIALS AND METHODS: We performed a prospective study of 71 consecutive patients that were submitted to MRI of the liver using a 1.5T magnet. The six following pulse sequences were used: 1) fat-suppressed respiratory triggered with conventional body coil; 2) breath-hold fat-suppressed with conventional body coil; 3) non-suppressed respiratory triggered with conventional body coil; 4) breath-hold non fat-suppressed with conventional body coil; 5) fat-suppressed respiratory triggered with phased-array multicoil; 6) breath-hold fat-suppressed with phased-array multicoil. Images were analyzed quantitatively by measuring the signal-to-noise ratios and qualitatively by evaluating the sharpness of hepatic contours, visibility of intrahepatic vessels and other segmental landmarks, and the presence of artifacts. RESULTS: The qualitative analysis showed that the mean values obtained with the six sequences were 7.8, 4.6, 7.9, 5.2, 6.7 and 4.6, respectively. The respiratory-triggered sequences were better than the breath-hold sequences in both qualitative and quantitative analysis $(p<0.001)$. No significant differences in the values of signal-to-noise ratios and in overall image quality rankings were found between the sequences with and without fat suppression ( $p>0.05$ ). The sequences using the body coil were similar in terms of image quality ( $p>0.05$ ) and better regarding signal-to-noise ratios than those obtained with the phased-array multicoil ( $<<0.001$ ). CONCLUSION: Our qualitative and quantitative results suggest that the best MRI sequences for the evaluation of the liver are the sequences with respiratory triggering using a conventional body coil, with or without fat suppression.

Key words: Magnetic resonance imaging; Liver; Gastrointestinal diagnostic techniques; Imaging techniques.

\footnotetext{
* Trabalho realizado no Departamento de Diagnóstico por Imagem da Universidade Federal de São Paulo/Escola Paulista de Medicina (Unifesp/EPM), São Paulo, SP.

1. Pós-graduandas do Departamento de Diagnóstico por Imagem da Unifesp/EPM.
}

2. Professor Adjunto do Departamento de Diagnóstico por Imagem da Unifesp/EPM.

3. Professor Livre-Docente, Chefe do Departamento de Diagnóstico por Imagem da Unifesp/EPM.

Endereço para correspondência: Dra. Cristiane L. Abbehusen.
Rua Primitivo Moacir, 48, Itaigara. Salvador, BA, 41815-100. E-mail: cabbehusen@hotmail.com

Recebido para publicação em 5/11/2002. Aceito, após revisão, em 3/4/2003. 


\section{INTRODUÇÃO}

Os primeiros relatos de imagens de ressonância magnética (RM) com resolução e capacidade diagnósticas foram feitos em estudos do sistema nervoso central ${ }^{(\mathbf{1})}$. Os trabalhos iniciais, voltados para a avaliação do fígado pelaa RM, demonstravam imagens degradadas pelos artefatos de movimento fisiológico, resultando em baixa resolução espacial e reduzido poder diagnóstico ${ }^{(2)}$. Desde então, a pesquisa científica promoveu vários progressos tecnológicos que melhoraram substancialmente a qualidade das imagens abdominais pela RM. Entre estes progressos, destaca-se o surgimento de equipamentos com gradientes mais potentes que permitiram a criação de seqüências de pulso mais rápidas, reduziram o tempo de exame e minimizaram os artefatos de movimentos respiratórios e peristálticos. Neste cenário, surgiram também as bobinas de sinergia ("phased-array"), com melhora da relação sinal/ruído e da qualidade das imagens obtidas ${ }^{(3,4)}$.

Nos estudos de RM do fígado, as imagens ponderadas em T2 são fundamentais para a detecção e caracterização de lesões hepáticas focais, para a diferenciação entre tumores benignos e malignos, e para a avaliação de doenças hepáticas difusas, entre outras aplicações ${ }^{(5)}$. Inicialmente, as imagens ponderadas em T2 eram obtidas utilizando-se a sequiência "spin-echo" (SE) convencional, com tempo de aquisição freqüentemente superior a dez minutos. Estes longos tempos de aquisição foram provavelmente o principal responsável pelas limitações da RM do abdome - relacionadas à degradação da imagem pelos artefatos de movimentos fisiológicos e à redução da relação sinal/ruído ${ }^{(6)}$.

Com o surgimento de técnicas rápidas, como a "rapid acquisition with relaxation enhancement" (RARE) e suas derivadas "half-Fourier single-shot" (HASTE) e "turbo spin-echo" (TSE) ou "fast spin-echo" (FSE), a principal limitação das sequiências ponderadas em T2 foi contornada, obtendo-se imagens em tempos mais curtos, com baixa ocorrência de artefatos e mantendo uma elevada resolução espacial ${ }^{(7,8)}$. A resolução espacial de uma imagem é definida como a capacidade de se distinguir com precisão dois pontos contíguos ${ }^{(\mathbf{9})}$.
Estudos mais recentes têm demonstrado uma superioridade ainda maior das sequiências rápidas ponderadas em $\mathrm{T} 2$, quando associadas à técnica de supressão de gordura. Nas imagens ponderadas em T2, a adição da supressão de gordura, particularmente nas seqüências TSE, pode aumentar a detecção de lesões hepáticas focais por reduzir o sinal hepático de fundo (melhora da relação contraste/ruído) e os artefatos-fantasma ou "ghost artifacts"(10). Por outro lado, a manutenção da elevada intensidade de sinal da gordura é útil para a delimitação anatômica dos contornos de órgãos parenquimatosos, para a avaliação de disseminação local de tumores, e para o auxílio na caracterização de massas com componente lipídico ${ }^{(\mathbf{1 1 1})}$. Devido a estas considerações, entre outras, encontram-se, na literatura, resultados conflitantes à respeito da utilização da técnica de supressão de gordura, na rotina de obtenção de sequiências rápidas ponderadas em $\mathrm{T} 2$, para o estudo do abdome através da RM $^{(12-17)}$.

Existem diversas técnicas para reduzir ou eliminar os artefatos provenientes da respiração em estudos de RM do abdome superior. Entre estas, uma das mais utilizadas é a aplicação de sincronização respiratória, que consiste em adquirir as imagens apenas durante a expiração, quando o movimento respiratório tem menor amplitude, utilizando uma faixa abdominal e um dispositivo eletrônico para detecção de movimentos, o sincronizador respiratório. Esta técnica reduz os artefatos provenientes da respiração, mas aumenta o tempo de aquisição, em função da frequiência e do ritmo respiratório do paciente ${ }^{(9)}$.

Apesar dos progressos na resolução temporal e espacial das imagens abdominais ponderadas em $\mathrm{T} 2$, utilizando sequiências rápidas e sincronizador respiratório, o tempo de aquisição destas seqüências é frequientemente superior a dois minutos e não permite sequiências em apnéia, que poderiam potencialmente eliminar por completo os artefatos de respiração. Com esta finalidade, foram adotadas técnicas mais rápidas de aquisição de imagem, que permitiram realizar as sequiências ponderadas em T2, durante o curso de uma única apnéia, eliminando, teoricamente, os artefatos de respiração e melhorando, assim, a qualidade das imagens obtidas ${ }^{(\mathbf{1 8 , 1 9 )}}$.
Apesar das evidentes vantagens das sequiências em apnéia, os estudos existentes comparando esta técnica com aquela que utiliza a SR ainda são controversos quanto à melhora da qualidade da imagem e da sua eficiência diagnóstica ${ }^{(18-20)}$.

Os exames de RM do fígado foram tradicionalmente realizados com a bobina de corpo inteiro inserida no próprio equipamento (bobina de quadratura). Há alguns anos, surgiram as bobinas de sinergia ou "phased-array", com aplicação inicial em estudos de coluna vertebral e, mais recentemente, também em exames de abdome e pelve ${ }^{(21,22)}$. A bobina de sinergia é uma bobina de superfície constituída de um arranjo de pares de bobinas de quadratura destinadas à recepção simultânea de distintos sinais de RM. Estas bobinas são posicionadas em contato com a parede abdominal do paciente, obtendo-se, desta forma, imagens com elevada relação sinal/ruído, mantendo-se amplo campo de visão ${ }^{(23)}$.

Apesar de diversos autores terem demonstrado a superioridade da bobina de sinergia no estudo abdominal, estes trabalhos foram realizados com equipamentos diferentes daquele à nossa disposição, que poderia apresentar resultados distintos ${ }^{(24)}$. Deve-se considerar, também, que as despesas de aquisição de um equipamento com bobina de sinergia são significativamente superiores, e eventualmente dispensáveis, se a melhora da qualidade diagnóstica das imagens obtidas não for substancial ${ }^{(23,24)}$.

Idealmente, as imagens de RM deveriam apresentar elevada resolução espacial, alta relação sinal/ruído, e serem obtidas em tempos curtos, para evitar a ocorrência de artefatos de movimentos e permitir excelente eficácia diagnóstica. No entanto, após revisão bibliográfica, percebemos que não existe pleno consenso na adoção da melhor técnica de aquisição de imagens ponderadas em T2, para estudos do fígado, principalmente no que se refere ao uso de apnéia, sincronização respiratória e supressão de gordura. A eficiência destes recursos pode depender, entre outros fatores, do tipo de equipamento utilizado.

O objetivo deste trabalho foi comparar, qualitativamente e quantitativamente, as sequiências rápidas (TSE) para a obtenção de imagens ponderadas em T2, utilizandose a sincronização respiratória, apnéia, su- 
pressão de gordura e bobina de sinergia, na avaliação do fígado pela RM.

\section{MATERIAIS E MÉTODOS}

\section{Casuística}

Entre abril e dezembro de 2000, foi realizado estudo prospectivo em 71 pacientes consecutivos submetidos a exames de RM do fígado.

Destes 71 pacientes, 34 eram do sexo feminino $(47,9 \%)$ e 37 , do sexo masculino (52,1\%), com idade média de 40,7 anos, variando de 15 a 82 anos (Tabela 1).

Todos os pacientes foram informados e orientados sobre como seria realizado o exame de RM e assinaram o termo de consentimento para poderem participar de protocolo de pesquisa, previamente analisado e aprovado pelo Comitê de Ética em Pesquisa desta Instituição.

Todos os pacientes encaminhados para realização de exame de RM do abdome superior foram admitidos no estudo independentemente da idade, indicação do exame, doença de base, ritmo respiratório ou capacidade de manter a apnéia. Os únicos critérios de exclusão foram a necessidade de uso de sedação anestésica para realizar o exame (em pacientes claustrofóbicos, por exemplo) e casos de pacientes inconscientes ou incapazes de obedecer comandos.

Foram realizadas 402 seqüências ponderadas em T2, com uma média de 5,66 sequiências por paciente. Em 10/71 pacientes $(14 \%)$ não foi possível realizar todas as seis seqüências propostas, em virtude de dificuldades técnicas ou limitação do próprio paciente (por exemplo, cansaço, desconforto, etc.).

\section{Técnica de exame}

Os exames foram executados em aparelho da marca Philips, modelo ACS NT15, operando em alto campo magnético $(1,5 \mathrm{~T})$ e equipado com gradientes de $15 \mathrm{mT} / \mathrm{s}$.

Foram realizadas seis sequiências TSE ponderadas em T2: 1) supressão de gordura (SPIR) com sincronização respiratória (SR) e bobina de corpo inteiro (BC); 2) supressão de gordura em apnéia (AP) e bobina de corpo inteiro; 3) sem supressão de gordura com sincronização respiratória e bobina de corpo inteiro; 4) sem supressão de gordura em apnéia e bobina de cor-
Tabela 1 Distribuição dos pacientes segundo a faixa etária.

\begin{tabular}{|c|c|c|}
\hline $\begin{array}{c}\text { Idade } \\
\text { (anos) }\end{array}$ & $\begin{array}{c}\text { № de } \\
\text { pacientes }\end{array}$ & Porcentagem \\
\hline $15-30$ & 10 & $14,1 \%$ \\
$31-45$ & 21 & $29,6 \%$ \\
$46-60$ & 23 & $32,4 \%$ \\
$61-90$ & 17 & $23,9 \%$ \\
\hline Total & 71 & $100 \%$ \\
\hline
\end{tabular}

po inteiro; 5) com supressão de gordura com sincronização respiratória e bobina de sinergia (BS); 6) com supressão de gordura em apnéia e bobina de sinergia.

Todas as seqüências TSE ponderadas em T2 foram realizadas com tempo de eco de 90 milissegundos. Procuramos, também, manter constantes outros importantes parâmetros técnicos, como tempo de repetição, espessura de corte, número de cortes, campo de visão e matriz de aquisição (Quadro 1). Foi necessário modificar ligeiramente o TR, o número de cortes e o número de aquisições, nas seqüências em apnéia, para manter os tempos de aquisição dentro de limites aceitáveis (inferiores a 30 segundos).

Em todos os pacientes utilizou-se faixa de compressão abdominal, para reduzir os artefatos de respiração, e injeção de antiespasmódico (Buscopan ${ }^{\circledR}, 40 \mathrm{mg}$, via endovenosa), imediatamente antes do início do exame, para reduzir os artefatos causados pelos movimentos peristálticos.

\section{Interpretação dos exames de ressonância magnética}

Inicialmente, todas as seqüências realizadas foram comparadas conjuntamente, através de parâmetros qualitativos e quantitativos, sendo realizada uma graduação em ordem decrescente das melhores para as piores seqüências. Posteriormente, foram comparadas as seqüências pareadas, que diferiam apenas em um parâmetro do estudo, com o objetivo de avaliar separadamente técnica de controle respiratório, utilização de supressão de gordura e tipo de bobina de radiofrequiência.

\section{a) Avaliação qualitativa}

A avaliação qualitativa das imagens foi realizada de forma independente por dois radiologistas com experiência em diagnóstico por imagem do fígado. As seis sequiências de um mesmo paciente foram analisadas, simultânea e comparativamente, por cada um dos observadores, que desconheciam o tipo de seqüência em estudo.

Segundo metodologia empregada em outros trabalhos da literatura, foram utilizados três critérios na avaliação da qualidade das imagens: 1) detecção de estruturas de referência anatômica intra-hepáticas; 2) definição dos contornos hepáticos; 3) presença de artefatos de respiração ${ }^{(\mathbf{1 8 , 1 9 )}}$.

As estruturas de referência anatômica avaliadas foram a vesícula biliar, a veia cava inferior, a veia porta, as veias hepáticas, o ligamento falciforme e a artéria hepática

Quadro 1 Parâmetros técnicos utilizados nas seis seqüências realizadas.

\begin{tabular}{|l|c|c|c|c|c|c|}
\hline \multirow{2}{*}{ Parâmetro } & \multicolumn{7}{c|}{ Seqüência } \\
\cline { 2 - 7 } & SPIR/SR/BC & SPIR/AP/BC & S/SPIR/SR/BC & S/SPIR/AP/BC & SPIR/SR/BS & SPIR/AP/BS \\
\hline TR (ms) & 1.800 & 2.100 & 1.800 & 1.800 & 1.800 & 2.100 \\
TE (ms) & 90 & 90 & 90 & 90 & 90 & 90 \\
FOV & 400 & 400 & 400 & 400 & 400 & 400 \\
RFOV (\%) & 80 & 75 & 80 & 75 & 80 & 75 \\
Matriz & 256 & 256 & 256 & 256 & 256 & 256 \\
Reconstrução & 512 & 512 & 512 & 512 & 512 & 512 \\
Número de cortes & 24 & 20 & 24 & 24 & 24 & 20 \\
Espessura (mm) & 8 & 8 & 8 & 8 & 8 & 8 \\
"Gap" & 0,8 & 0,8 & 0,8 & 0,8 & 0,8 & 0,8 \\
"Half scan" & Não & Não & Não & Não & Não & Não \\
"Turbo factor" & 18 & 19 & 18 & 19 & 18 & 19 \\
NSA & 2 & 1 & 2 & 1 & 2 & 1 \\
Tempo de aquisição & 148 & 25 & 148 & 24 & 148 & 25 \\
\hline
\end{tabular}

SPIR: com supressão de gordura; s/SPIR: sem supressão de gordura; SR: sincronização respiratória; AP: apnéia; BC: bobina de corpo; BS: bobina de sinergia; TR: tempo de repetição; TE: tempo de eco; FOV: "field of view"; NSA: "number of signal acquired"; ms: milissegundos; mm: milímetro(s). 
comum. Para este critério, foram dados pontos de 1 a 3 , de acordo com o número de estruturas de referência anatômica identificadas na imagem. Um ponto foi dado quando até duas estruturas de referência anatômica foram identificadas; dois pontos, quando de três a quatro; e três pontos, quando de cinco a seis estruturas de referência foram observadas. Foram selecionados estes pontos de referência anatômica, em virtude de serem aqueles utilizados na segmentação hepática ${ }^{(\mathbf{2 5 , 2 6})}$ (Figura 1).

A definição dos contornos hepáticos foi pontuada também de um a três. Foi atri-

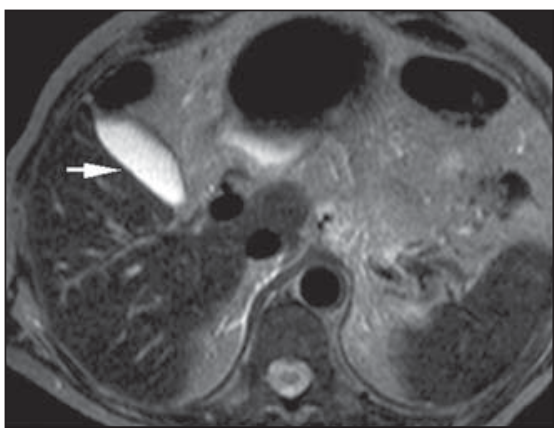

A

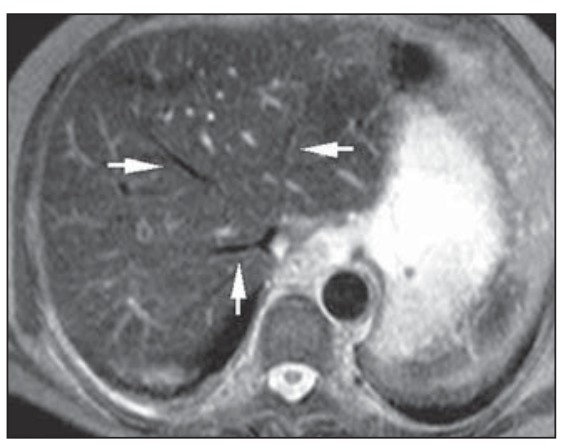

D

Figura 1. Demonstração dos parâmetros anatômicos utilizados na análise qualitativa. A: vesícula biliar (seta); B: veia cava inferior (seta); C: veia porta (seta); D: veias hepáticas (setas); E: ligamento falciforme (setas); F: artéria hepática comum (seta).

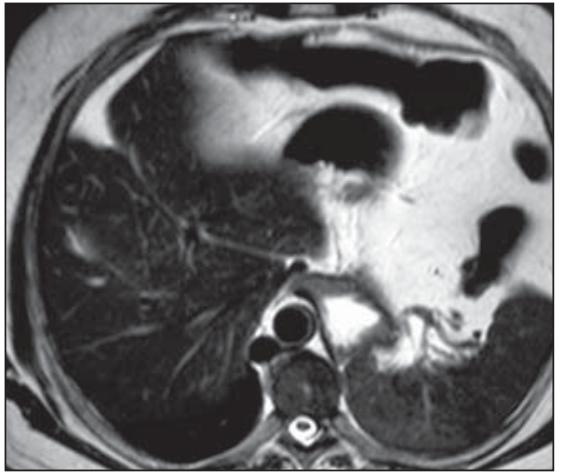

A buído um ponto, quando os contornos eram mal definidos; dois pontos, quando se caracterizava uma boa definição dos contornos; e três pontos, na presença de uma excelente definição dos contornos hepáticos (Figura 2).

Os artefatos de respiração foram também pontuados de um a três: um ponto, quando presentes e afetando o diagnóstico; dois pontos, quando presentes, sem afetar o diagnóstico; e três pontos, quando ausentes (Figura 3).

Posteriormente, foi somada a pontuação obtida com os três critérios e foi cal-

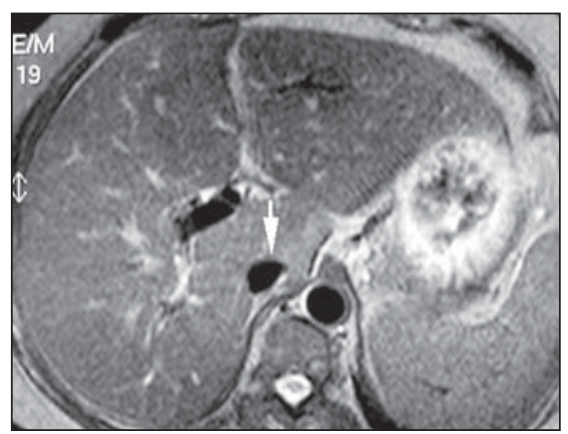

B

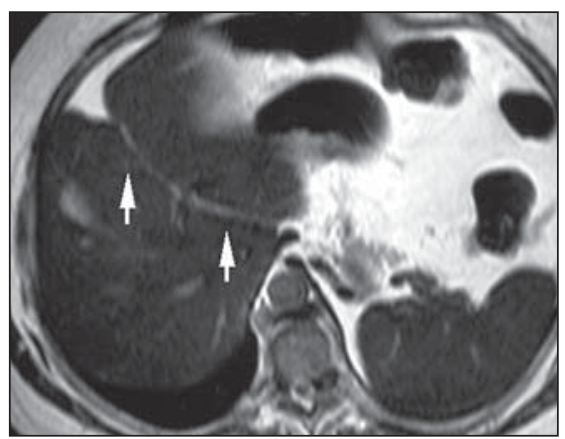

$\mathbf{E}$ culado um índice global da qualidade da imagem, variando de 3 a 9, que foi utilizado para análise comparativa dos parâmetros qualitativos das diversas sequiências ponderadas em $\mathrm{T} 2$.

\section{b) Avaliação quantitativa}

Utilizando-se metodologia adotada por outros autores $^{(\mathbf{1 8 , 1 9 )}}$, foram mensuradas as intensidades de sinal do fígado e do ruído de fundo, em uma mesma região, em todas as sequiências de todos os pacientes, por um único radiologista, em uma estação de trabalho independente.

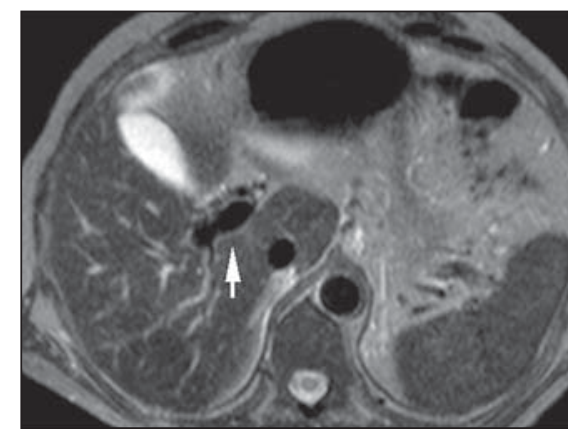

C

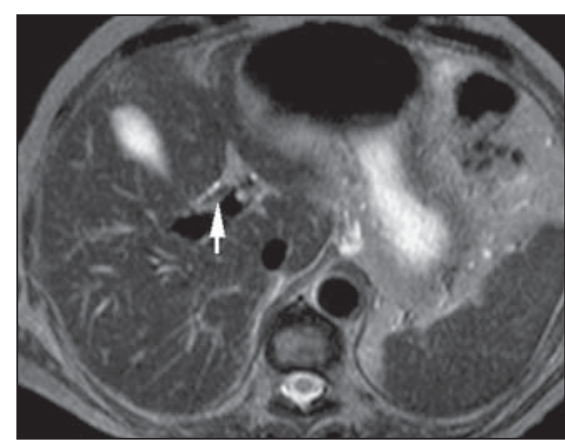

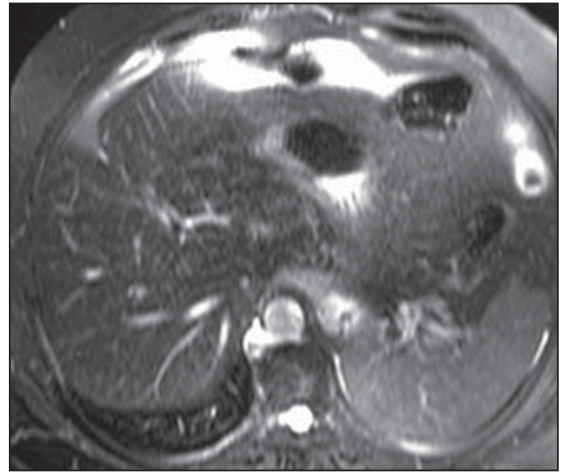

B

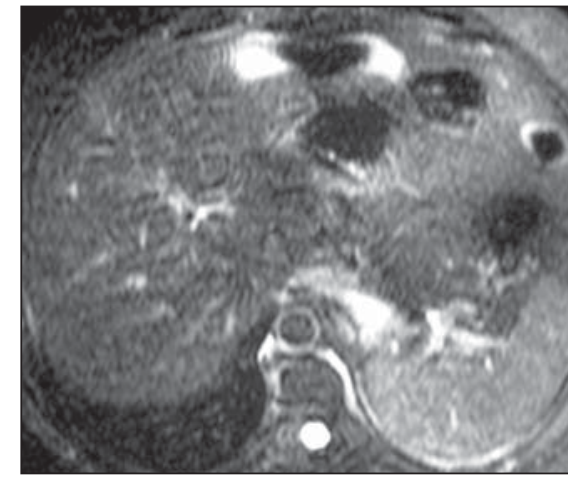

C

Figura 2. Exemplificação da graduação qualitativa quanto à definição dos contornos do fígado. A: excelente definição (nota 3); B: boa definição (nota 2); C: má definição (nota 1). 


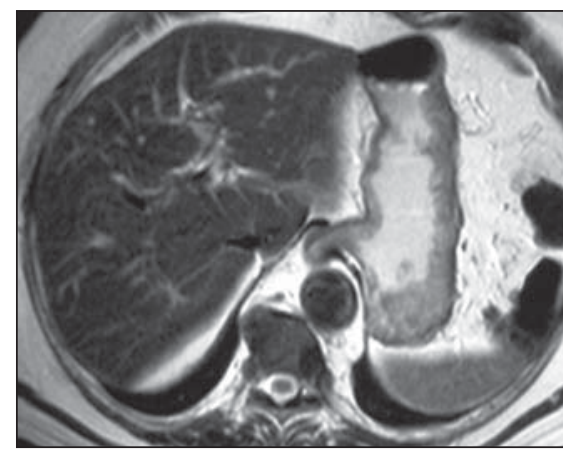

A

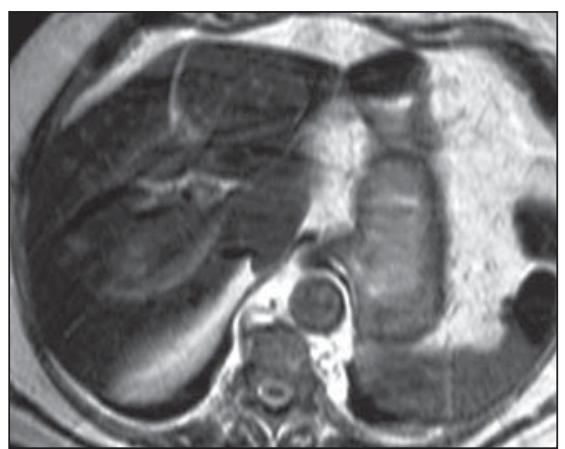

B

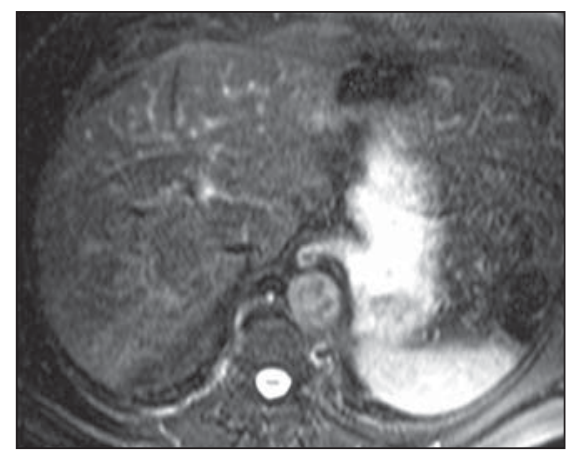

C

Figura 3. Exemplificação da graduação qualitativa quanto à presença de artefatos de respiração. A: artefatos ausentes (nota 3); B: artefatos presentes sem afetar o diagnóstico (nota 2); c: artefatos presentes e afetando o diagnóstico (nota 1).

As intensidades de sinal foram obtidas através da colocação manual de um ROI, em locais previamente estabelecidos: no lobo hepático direito e adjacente à parede abdominal anterior direita, para a mensuração do ruído de fundo (Figura 4). Evitamos colocar o ROI em região contendo grandes vasos hepáticos, para não contaminar a leitura do sinal proveniente do parênquima com aquele emitido pelo fluxo vascular. Para todas as medidas, o tamanho do ROI foi idêntico.

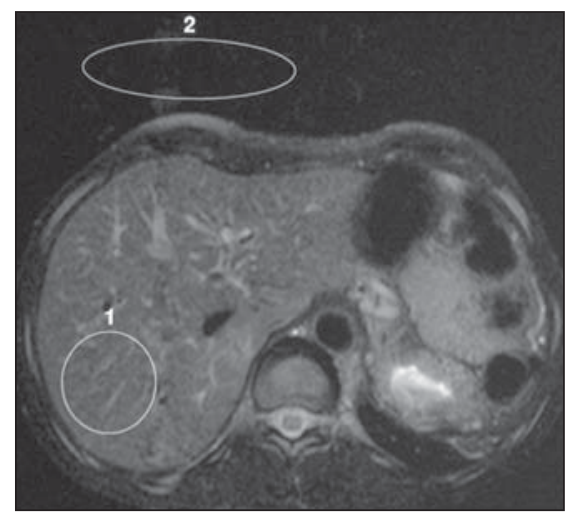

Figura 4. Exemplificação do local utilizado e das dimensões do ROI para mensuração das intensidades de sinal do fígado (1) e do ruído de fundo (2).

Seguindo parâmetros aceitos na literatura, a relação da intensidade de sinal do fígado e do ruído de fundo foi quantificada pela fórmula $\mathrm{IS}_{\mathrm{F}} / \mathrm{R}$, onde $\mathrm{IS}_{\mathrm{F}}$ representa a intensidade de sinal do fígado, e $\mathrm{R}$ a do ruído de fundo ${ }^{(18,19)}$

\section{Método estatístico}

\section{a) Concordância interobservador}

Para o cálculo da concordância interobservador na avaliação qualitativa, tanto global quanto por sequiência, após preenchida a ficha com o valor obtido para o índice global da qualidade da imagem em cada seqüência realizada, para cada um dos observadores, foram realizadas duas tabelas adicionais. Na primeira tabela, as pontuações foram subdivididas em três grupos: notas 3 e 4 foram agrupadas como qualidade ruim; notas 5 e 6 , como qualidade regular; e notas 7 a 9, como boa qualidade de imagem. Posteriormente, foi criada uma terceira tabela utilizando-se apenas o critério da presença de concordância. A análise desta concordância foi categorizada através do índice Kappa ${ }^{(27)}$. Não foi possível realizar análise estatística para identificar a ou as seqüências mais reprodutíveis porque elas, globalmente, diferiam em mais de um parâmetro técnico.

\section{b) Análises qualitativa e quantitativa}

Para os estudos qualitativo e quantitativo das sequiências, utilizamos a média das notas de dois examinadores como escore creditado a cada grupo. Depois, comparamos as notas das seis sequiências, pelo teste de Kruskal-Wallis, por se tratar de comparações de múltiplos grupos, em amostra que não apresentava distribuição compatível com a curva normal. Após constatação da significante diferença global, realizamos comparações múltiplas com auxílio do pós-teste de Dunn, para identificar quais os grupos com maiores escores. Por fim, utilizamos o teste de Mann-Whitney para avaliar as variáveis que se diferenciaram entre duas seqüências específicas.

As análises foram realizadas com auxílio do "software" SPSS 11.0 (Statistical Package for Social Sciences) e foram con- siderados como significativos valores de $\mathrm{p}$ inferiores a 5\% ( $\mathrm{p}<0,05)$. Quando a estatística calculada foi significante, usamos um asterisco $(*)$ para caracterizá-la. Caso contrário, usamos N.S. (não-significante).

\section{RESULTADOS}

\section{Concordância interobservador}

Na Figura 5 observa-se a concordância interobservador na avaliação qualitativa das imagens, para cada sequiência realizada. A concordância global entre os dois observadores para a seqüência SPIR/SR/ BC foi de $81,7 \%$; para a SPIR/AP/BC, de 70,6\%; para a s/SPIR/SR/BC, de 91,5\%; para a s/SPIR/AP/BC, de 66,7\%; para a SPIR/SR/BS, de 67,2\%; e para a SPIR/AP/ BS, de $77,4 \%$.

Segundo o índice Kappa ${ }^{(27)}$, o nível de concordância interobservador foi considerado substancial para as sequiências SPIR/ AP/BC, s/SPIR/AP/BC, SPIR/SR/BS e SPIR/AP/BS e quase perfeito para SPIR/ $\mathrm{SR} / \mathrm{BC}$ e s/SPIR/SR/BC.

\section{Análise qualitativa}

O valor médio do índice global da qualidade das imagens analisadas pelos dois observadores, obtido em cada seqüência, e o desvio-padrão foram, respectivamente, $7,8 \pm 1,0$ para a seqüência SPIR/SR/BC, 4,6 $\pm 0,9$ para a seqüência SPIR/AP/BC, $8,0 \pm 0,9$ para a seqüência s/SPIR/SR/BC, $5,3 \pm 1,2$ para a seqüência s/SPIR/AP/BC, $6,7 \pm 1,1$ para a seqüência $S P I R / S R / B S$, e $4,7 \pm 1,0$ para a seqüência SPIR/AP/BS (Tabela 2).

Aplicando o teste estatístico de Kruskal-Wallis, obteve-se uma graduação das 


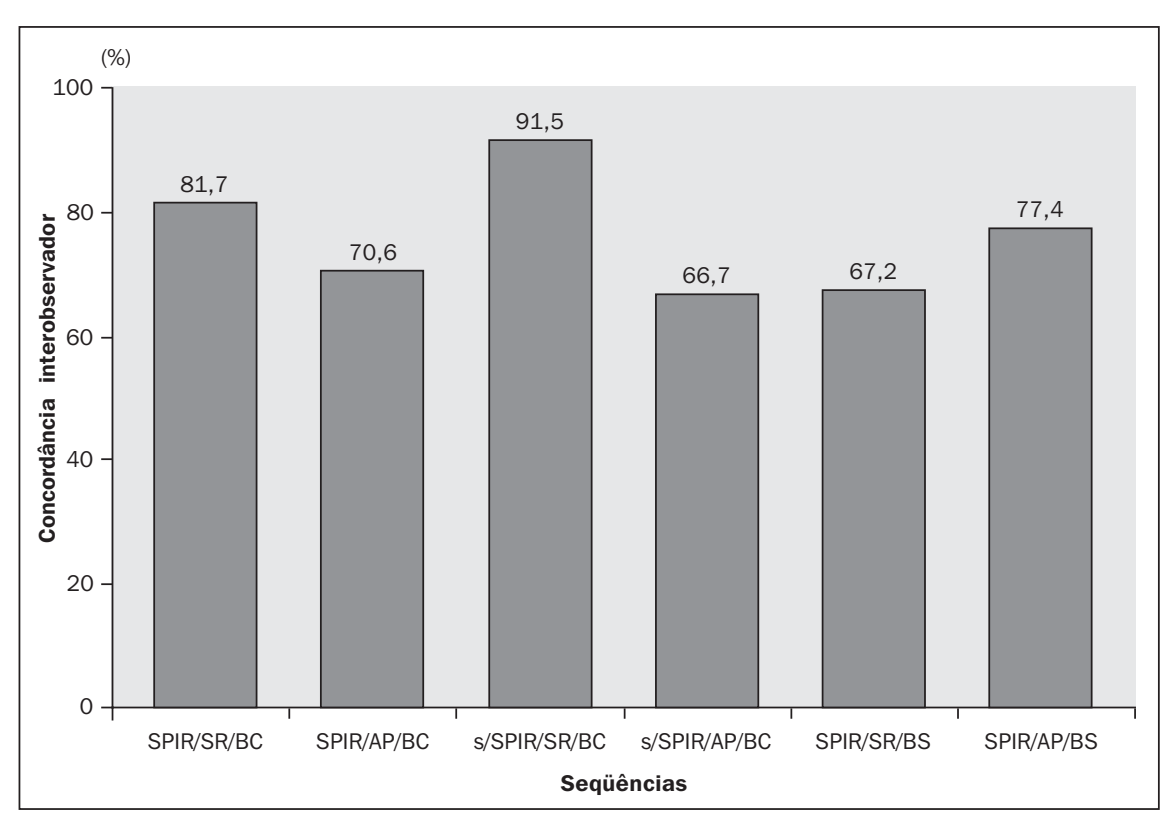

Figura 5. Gráfico demonstrativo da concordância global interobsenvador para cada seqüência realizada. (SPIR: com supressão de gordura; s/SPIR: sem supressão de gordura; SR: sincronização respiratória; AP: apnéia; BC: bobina de corpo; BS: bobina de sinergia).

Tabela 2 Valor médio do índice global da qualidade das imagens para cada seqüência e desvio-padrão (DP).

\begin{tabular}{|c|c|}
\hline Seqüência & Valor médio \pm DP \\
\hline SPIR/SR/BC & $7,8 \pm 1,0$ \\
SPIR/AP/BC & $4,6 \pm 0,9$ \\
S/SPIR/SR/BC & $8,0 \pm 0,9$ \\
S/SPIR/AP/BC & $5,3 \pm 1,2$ \\
SPIR/SR/BS & $6,7 \pm 1,1$ \\
SPIR/AP/BS & $4,7 \pm 1,1$ \\
\hline
\end{tabular}

SPIR: com supressão de gordura; s/SPIR: sem supressão de gordura; SR: sincronização respiratória; AP: apnéia; BC: bobina de corpo; BS: bobina de sinergia.

melhores seqüências, em ordem decrescente, segundo a análise qualitativa: s/SPIR/SR/ BC, SPIR/SR/BC, SPIR/SR/BS, s/SPIR/ AP/BC, SPIR/AP/BS e SPIR/AP/BC.

Quando foram realizadas comparações múltiplas, as seqüências SPIR/SR/BC e s/SPIR/SR/BC continuaram graduadas como as melhores seqüências pela análise qualitativa das imagens, não havendo diferença estatisticamente significativa entre elas $(\mathrm{p}=1,0)$. Ambas foram superiores à sequiência SPIR/SR/BS ( $p<0,001$ ), que foi melhor, por sua vez, que a seqüência s/SPIR/AP/BC, com valor estatisticamente significante $(\mathrm{p}<0,001)$. Esta última foi superior à SPIR/AP/BS, com significância estatística $(\mathrm{p}<0,001)$, embora não tenha havido diferença estatisticamente significa- tiva entre ela e a sequiência SPIR/AP/BC $(\mathrm{p}=1,0)$ (Tabela 3).

\section{Análise quantitativa}

A média dos valores encontrados entre a relação da intensidade de sinal do fígado e a da intensidade de sinal do ruído de fundo, em cada seqüência realizada, foi de 13,6 para a seqüência SPIR/SR/BC, 11,1 para a seqüência SPIR/AP/BC, 11,4 para

Tabela 3 Comparações múltiplas dos resultados obtidos através da análise qualitativa das imagens entre as diversas seqüências realizadas.

\begin{tabular}{|ll|c|c|}
\hline \multicolumn{2}{|c|}{ Comparações entre as seqüências } & Valor médio & $p$ \\
\hline SPIR/SR/BC & SPIR/AP/BC & $4,6 \pm 0,9$ & $<0,001^{*}$ \\
$7,8 \pm 1,0$ & S/SPIR/SR/BC & $8,0 \pm 0,9$ & $<1,0$ (N.S.) \\
& S/SPIR/AP/BC & $5,3 \pm 1,2$ & $<0,001^{*}$ \\
& SPIR/SR/BS & $6,7 \pm 1,1$ & $<0,001^{*}$ \\
& SPIR/AP/BS & $4,7 \pm 1,1$ & $<0,001^{*}$ \\
SPIR/AP/BC & S/SPIR/SR/BC & $8,0 \pm 0,9$ & $<0,001^{*}$ \\
$4,6 \pm 0,9$ & S/SPIR/AP/BC & $5,3 \pm 1,2$ & $<0,001^{*}$ \\
& SPIR/SR/BS & $6,7 \pm 1,1$ & $<0,001^{*}$ \\
& SPIR/AP/BS & $4,7 \pm 1,1$ & $=1,0(\mathrm{~N} . S)$. \\
s/SPIR/SR/BC & S/SPIR/AP/BC & $5,3 \pm 1,2$ & $<0,001^{*}$ \\
$8,0 \pm 0,9$ & SPIR/SR/BS & $6,7 \pm 1,1$ & $<0,001^{*}$ \\
& SPIR/AP/BS & $4,7 \pm 1,1$ & $<0,001^{*}$ \\
S/SPIR/AP/BC & SPIR/SR/BS & $6,7 \pm 1,1$ & $<0,001^{*}$ \\
$5,3 \pm 1,2$ & SPIR/AP/BS & $4,7 \pm 1,1$ & $<0,001^{*}$ \\
SPIR/SR/BS & SPIR/AP/BS & $4,7 \pm 1,1$ & $<0,001^{*}$ \\
$6,7 \pm 1,1$ & & & \\
\hline
\end{tabular}

SPIR: com supressão de gordura; s/SPIR: sem supressão de gordura; SR: sincronização respiratória; AP: apnéia; BC: bobina de corpo; BS:bobina de sinergia; * significância estatística; (N.S.): não-significante. 
Tabela 4 Valor médio da relação sinal/ruído das imagens para cada seqüência e desvio-padrão (DP).

\begin{tabular}{|l|c|}
\hline Seqüência & Valor médio $\pm \mathrm{DP}$ \\
\hline $\mathrm{SPIR} / \mathrm{SR} / \mathrm{BC}$ & $13,6 \pm 8,4$ \\
$\mathrm{SPIR} / \mathrm{AP} / \mathrm{BC}$ & $11,1 \pm 5,1$ \\
S/SPIR/SR/BC & $11,4 \pm 4,2$ \\
$\mathrm{~S} / \mathrm{SPIR} / \mathrm{AP} / \mathrm{BC}$ & $7,3 \pm 3,9$ \\
$\mathrm{SPIR} / \mathrm{SR} / \mathrm{BS}$ & $6,5 \pm 3,2$ \\
$\mathrm{SPIR} / \mathrm{AP} / \mathrm{BS}$ & $5,5 \pm 2,7$ \\
\hline
\end{tabular}

SPIR: com supressão de gordura; s/SPIR: sem supressão de gordura; SR: sincronização respiratória; AP: apnéia; BC: bobina de corpo; BS: bobina de sinergia.

à técnica de controle da respiração, isto é, sincronização respiratória ou apnéia, em ambas utilizando-se bobina de corpo, observou-se superioridade qualitativa e quantitativa das sequiências realizadas com sincronização respiratória, independentemente da utilização ou não de técnica de supressão de gordura, com diferenças estatisticamente significantes $(\mathrm{p}<0,001)$ (Tabela 6 e Figuras 6 e 7).

\section{Análise isolada das sequiências diferenciadas apenas no que se refere à realização ou não de técnica de supressão de gordura}

A comparação isolada das seqüências realizadas com e sem supressão de gordura, especificamente utilizando bobina de corpo e sincronização respiratória, não demonstrou diferença estatisticamente significativa entre elas, tanto através da análise qualitativa quanto pela quantitativa, com $\mathrm{p}=1,0$ e $\mathrm{p}=0,5$, respectivamente (Tabela 6 e Figura 8). Quando a técnica de controle respiratório empregada foi a apnéia, observou-se superioridade da seqüêencia obtida sem supressão de gordura, do ponto de vista qualitativo, com significância estatística ( $\mathrm{p}<0,001$ ), não havendo diferença estatística significativa através da análise quantitativa das imagens adquiridas $(\mathrm{p}=0,07)$ (Tabela 6 e Figura 9).

\section{Análise isolada das sequiências diferenciadas apenas quanto à bobina de radiofreqüência utilizada}

Quando comparamos as seqüências pareadas, realizadas com sincronização respiratória e técnica de supressão de gordura, distintas apenas no tipo de bobina de radiofrequiência usada, notamos que as seqüências realizadas com bobina de corpo

Tabela 5 Comparações múltiplas dos resultados obtidos através da análise quantitativa das imagens entre as diversas seqüências realizadas.

\begin{tabular}{|ll|r|c|}
\hline \multicolumn{2}{|c|}{ Comparações entre as seqüências } & Valor médio & $p$ \\
\hline SPIR/SR/BC & SPIR/AP/BC & $11,1 \pm 5,1$ & 0,4 (N.S.) \\
$13,6 \pm 8,4$ & S/SPIR/SR/BC & $11,4 \pm 4,2$ & 0,5 (N.S.) \\
& S/SPIR/AP/BC & $7,3 \pm 3,9$ & $<0,001^{*}$ \\
& SPIR/SR/BS & $6,5 \pm 3,2$ & $<0,001^{*}$ \\
& SPIR/AP/BS & $5,5 \pm 2,7$ & $<0,001^{*}$ \\
SPIR/AP/BC & S/SPIR/SR/BC & $11,4 \pm 4,2$ & 1,0 (N.S.) \\
$11,1 \pm 5,1$ & S/SPIR/AP/BC & $7,3 \pm 3,9$ & 0,07 (N.S.) \\
& SPIR/SR/BS & $6,5 \pm 3,2$ & $<0,001^{*}$ \\
& SPIR/AP/BS & $5,5 \pm 2,7$ & $<0,001^{*}$ \\
S/SPIR/SR/BC & S/SPIR/AP/BC & $7,3 \pm 3,9$ & 0,05 (N.S.) \\
$10,0 \pm 4,2$ & SPIR/SR/BS & $6,5 \pm 3,2$ & $<0,001^{*}$ \\
& SPIR/AP/BS & $5,5 \pm 2,7$ & $<0,001^{*}$ \\
S/SPIR/AP/BC & SPIR/SR/BS & $6,5 \pm 3,2$ & 0,2 (N.S.) \\
$7,3 \pm 3,9$ & SPIR/AP/BS & $4,8 \pm 2,7$ & $<0,001^{*}$ \\
SPIR/SR/BS & SPIR/AP/BS & $5,5 \pm 2,7$ & 0,2 (N.S.) \\
$6,5 \pm 3,2$ & & & \\
\hline
\end{tabular}

SPIR: com supressão de gordura; s/SPIR: sem supressão de gordura; SR: sincronização respiratória; AP: apnéia; BC: bobina de corpo; BS: bobina de sinergia; * significância estatística; (N.S.): não-significante.

Tabela 6 Comparações isoladas entre as seqüências diferenciadas em apenas dois parâmetros.

\begin{tabular}{|l|c|c|c|}
\hline & Seqüência & Seqüência & $\mathrm{P}$ \\
\hline Controle dos artefatos de respiração & $\mathrm{SPIR} / \mathrm{SR} / \mathrm{BC}$ & $\mathrm{SPIR} / \mathrm{AP} / \mathrm{BC}$ & \\
Análise qualitativa & 7,8 & 4,6 & $<0,001^{*}$ \\
Análise quantitativa & 13,6 & 11,1 & $<0,001^{*}$ \\
& $\mathrm{~S} / \mathrm{SPIR} / \mathrm{SR} / \mathrm{BC}$ & $\mathrm{S} / \mathrm{SPIR} / \mathrm{AP} / \mathrm{BC}$ & \\
Análise qualitativa & 8,0 & 5,3 & $<0,001^{*}$ \\
Análise quantitativa & 11,4 & 8,8 & $<0,001^{*}$ \\
Supressão de gordura & $\mathrm{SPIR} / \mathrm{SR} / \mathrm{BC}$ & $\mathrm{S} / \mathrm{SPIR} / \mathrm{SR} / \mathrm{BC}$ & \\
Análise qualitativa & 7,8 & 8,0 & 1,0 (N.S.) \\
Análise quantitativa & 13,6 & 11,4 & 0,5 (N.S.) \\
& $\mathrm{SPIR} / \mathrm{AP} / \mathrm{BC}$ & $\mathrm{S} / \mathrm{SPIR} / \mathrm{AP} / \mathrm{BC}$ & \\
Análise qualitativa & 4,6 & 5,3 & $<0,001^{*}$ \\
Análise quantitativa & 11,1 & 8,8 & 0,07 (N.S.) \\
Bobina de radiofreqüência & $\mathrm{SPIR} / \mathrm{SR} / \mathrm{BC}$ & $\mathrm{SPIR} / \mathrm{SR} / \mathrm{BS}$ & \\
Análise qualitativa & 7,8 & 6,7 & $<0,001^{*}$ \\
Análise quantitativa & 13,6 & 7,3 & $<0,001^{*}$ \\
& $\mathrm{SPIR} / \mathrm{AP} / \mathrm{BC}$ & $\mathrm{SPIR} / \mathrm{AP} / \mathrm{BS}$ & \\
Análise qualitativa & 4,6 & 4,7 & 1,0 (N.S.) \\
Análise quantitativa & 11,1 & 5,5 & $<0,001^{*}$ \\
\hline
\end{tabular}

SPIR: com supressão de gordura; s/SPIR: sem supressão de gordura; SR: sincronização respiratória; AP: apnéia; BC: bobina de corpo; BS: bobina de sinergia; * significância estatística; (N.S.): não-significante.

apresentaram resultados superiores, tanto pela análise qualitativa quanto pela quantitativa ( $\mathrm{p}<0,001$ ) (Tabela 6 e Figura 10). As imagens obtidas com supressão de gordura e em apnéia não apresentaram diferença estatisticamente significante pela análise qualitativa $(\mathrm{p}=1,0)$, havendo uma superioridade da bobina de corpo pela avaliação quantitativa, com significância estatística (p < 0,001) (Tabela 6 e Figura 11).

\section{DISCUSSÃO}

Entre as inúmeras seqüências de RM disponíveis para a avaliação do fígado, as imagens ponderadas em T2 fornecem as mais importantes informações sobre detecção e caracterização de nódulos hepáti$\cos ^{(28)}$. A aquisição de imagens do fígado ponderadas em T2, com elevada resolução espacial e baixa resolução temporal, tem 


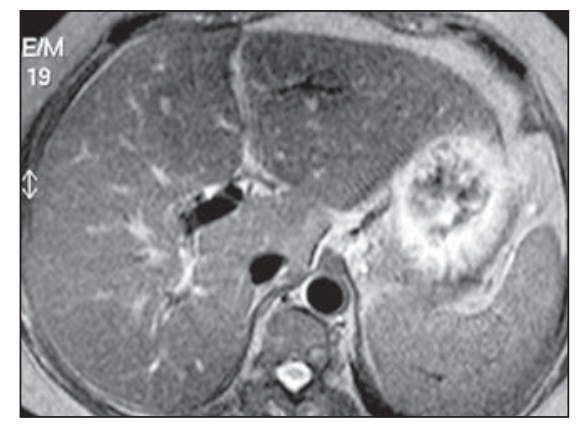

$\mathrm{SPIR} / \mathrm{SR} / \mathrm{BC}$

Nota $=9$ e IS $/$ $/ R=23,4$

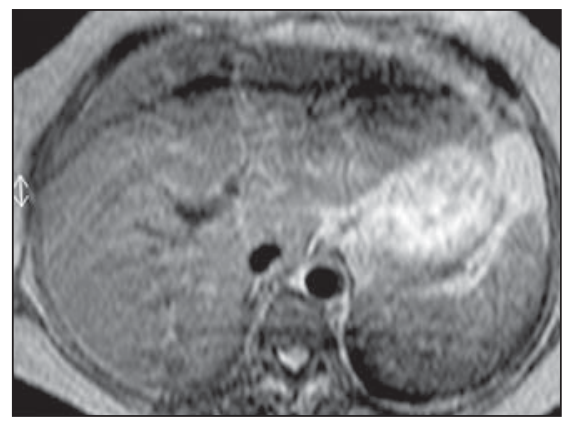

$\mathrm{SPIR} / \mathrm{AP} / \mathrm{BC}$

Nota $=3$ e IS $/ \mathrm{R}=11$

Figura 6. Exemplificação da avaliação isolada das seqüências pareadas diferenciadas quanto à técnica de controle da respiração, ambas com supressão de gordura (caso 21).

(SPIR: com supressão de gordura; SR: sincronização respiratória; AP: apnéia; BC: bobina de corpo; IS/R: intensidade de sinal do fígado/ruído de fundo).

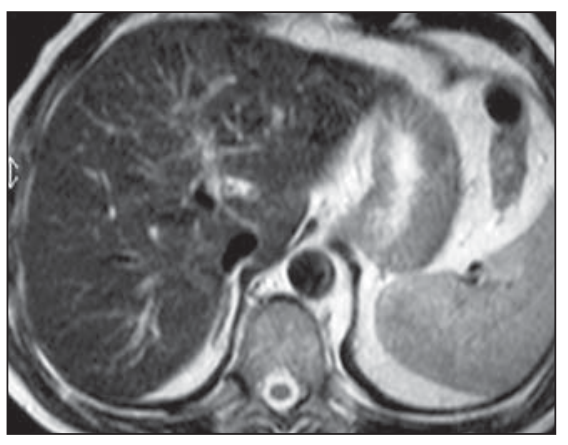

$\mathrm{S} / \mathrm{SPIR} / \mathrm{SR} / \mathrm{BC}$

Nota $=9$ e IS $/ \mathrm{R}=18$

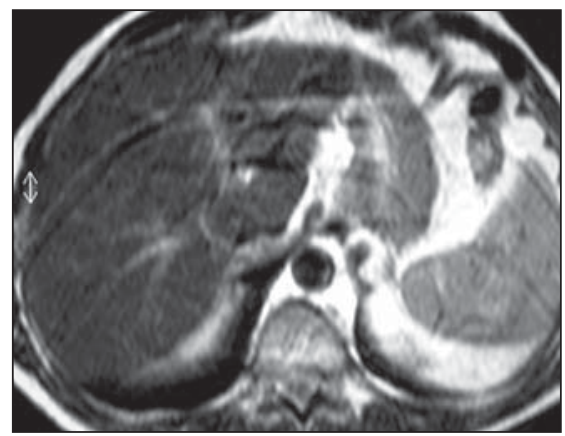

$\mathrm{S} / \mathrm{SPIR} / \mathrm{AP} / \mathrm{BC}$

Nota $=4$ e IS $/ \mathrm{R}=7,6$

Figura 7. Exemplificação da avaliação isolada das seqüências pareadas diferenciadas quanto à técnica de controle da respiração, ambas sem supressão de gordura (caso 25).

(SPIR: com supressão de gordura; s/SPIR: sem supressão de gordura; SR: sincronização respiratória; AP: apnéia; $\mathrm{BC}$ : bobina de corpo; IS $\mathrm{F} / \mathrm{R}$ : intensidade de sinal do fígado/ruído de fundo).

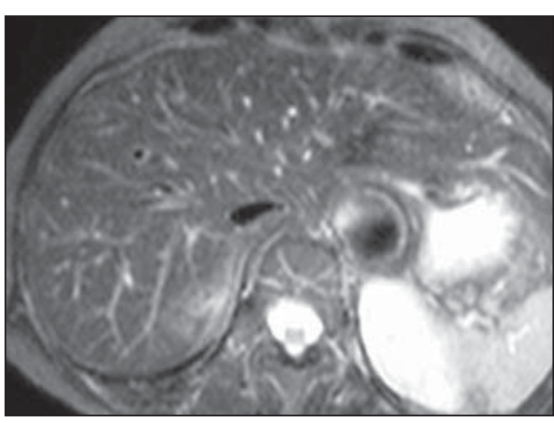

$\mathrm{SPIR} / \mathrm{SR} / \mathrm{BC}$

Nota $=9$ e IS $/ R=12,5$

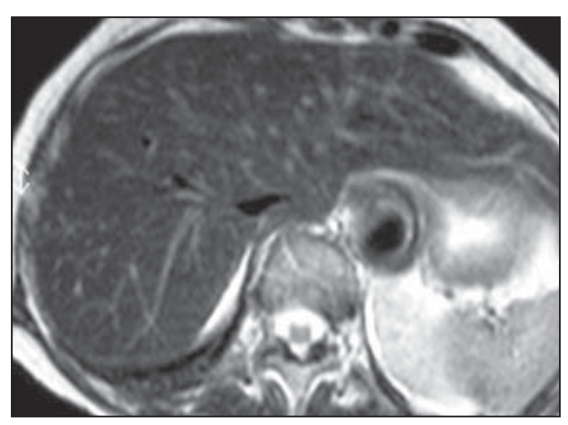

$\mathrm{S} / \mathrm{SPIR} / \mathrm{SR} / \mathrm{BC}$

Nota $=9$ e IS $/$ R $=10,1$

Figura 8. Exemplificação da avaliação isolada das seqüências pareadas diferenciadas quanto à utilização de supressão de gordura, ambas com sincronização respiratória (caso 13).

(SPIR: com supressão de gordura; s/SPIR: sem supressão de gordura; SR: sincronização respiratória; AP: apnéia; BC: bobina de corpo; IS $/$ R: intensidade de sinal do fígado/ruído de fundo).

sido, nos últimos anos, uma das principais áreas de interesse da investigação científica em técnicas de RM. Persiste ainda alguma controvérsia a respeito das técnicas a serem utilizadas, particularmente quanto ao uso de métodos para controle dos arte- fatos de respiração, supressão de gordura e tipo de bobina de radiofreqüência.

Muitos estudos realizaram a comparação isolada destes parâmetros técnicos, dificultando a identificação da melhor seqüência TSE ponderada em T2, a ser uti- lizada na prática clínica, principalmente considerando-se a necessidade de se combinarem os diversos recursos para a implementação de uma determinada seqüência com boa qualidade diagnóstica. Por esta razão, tentamos agrupar, em um único estudo, os principais pontos controversos existentes na literatura científica, com o intuito de determinar as melhores alternativas técnicas para obter imagens ponderadas em T2, que apresentem uma elevada qualidade de imagem e um alto poder diagnóstico nos exames de RM do fígado. Todas as sequiências deste estudo foram obtidas, na medida do possível, com os mesmos parâmetros técnicos (TE, TR, FOV, matriz, espessura de corte, etc.), diferindo apenas quanto à técnica de controle respiratório (sincronização respiratória ou apnéia), uso de supressão de gordura e o tipo de bobina de radiofrequiência (bobina de quadratura ou de sinergia).

Na revisão bibliográfica realizada, destaca-se também que nem todos os trabalhos foram prospectivos, com casuística às vezes restritas, heterogêneas e com parâmetros de exclusão que dificultam uma análise lúcida dos resultados apresentados ${ }^{(\mathbf{1 8 , 2 9 )}}$. É importante, também, observar que a quase totalidade desses estudos foi realizada com equipamentos das marcas General Electric e Siemens, ao contrário deste, que utilizou equipamento Philips. Os resultados obtidos a partir do uso de equipamentos de diversas procedências permitem reforçar a validação de certas práticas (como, por exemplo, a adoção preferencial de sequiências em apnéia) que poderiam estar relacionadas ao desenvolvimento técnico alcançado por determinados fabricantes, em direções específicas.

A concordância entre os dois observadores, através da análise qualitativa das imagens de todas as sequiências utilizadas neste estudo, demonstra a sua reprodutibilidade. O nível de concordância interobservador foi considerado substancial ou quase perfeito para todas as sequiências.

Um dos pontos mais controversos e avaliados em diversos trabalhos científicos é o método de controle dos artefatos de respiração. Várias técnicas foram propostas para suprimir estes artefatos, sendo a sincronização respiratória e a apnéia as

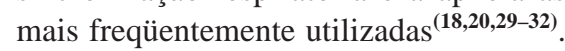




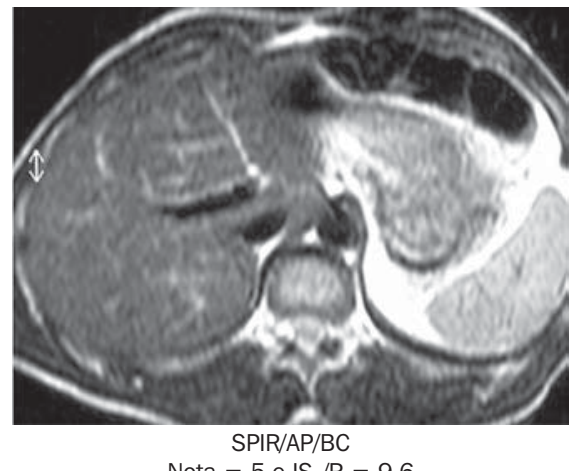

Nota $=5$ e IS $/$ R $=9,6$

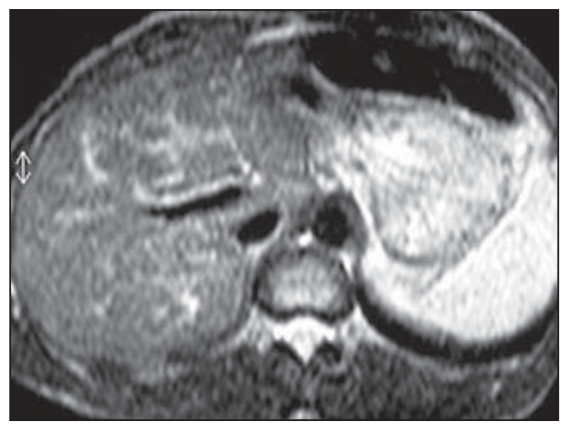

$\mathrm{S} / \mathrm{SPIR} / \mathrm{AP} / \mathrm{BC}$

Nota $=5$ e IS $/ \mathrm{R}=6,2$

Figura 9. Exemplificação da avaliação isolada das seqüências pareadas diferenciadas quanto à utilização de supressão de gordura, ambas em apnéia (caso 13).

(SPIR: com supressão de gordura; s/SPIR: sem supressão de gordura; SR: sincronização respiratória; AP: apnéia; BC: bobina de corpo; IS $/$ /R: intensidade de sinal do fígado/ruído de fundo).

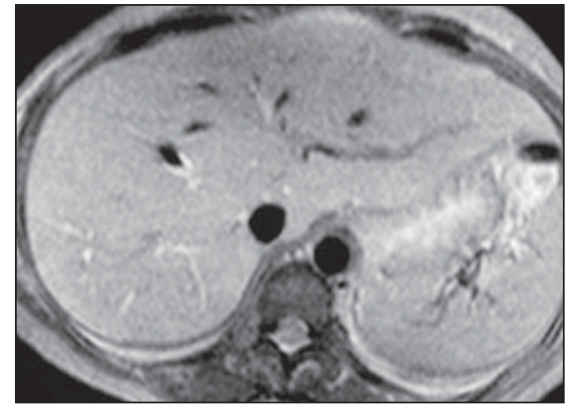

$\mathrm{SPIR} / \mathrm{SR} / \mathrm{BC}$

Nota $=9$ e IS $\mathrm{I}_{\mathrm{F}} / \mathrm{R}=26,6$

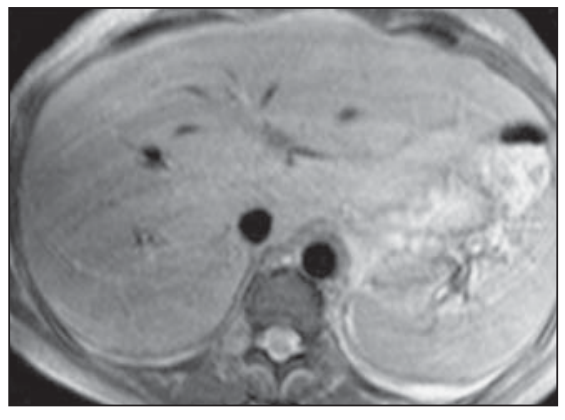

$\mathrm{SPIR} / \mathrm{SR} / \mathrm{BS}$

Nota $=5$ e IS $/$ F $=4,3$

Figura 10. Exemplificação da avaliação isolada das seqüências pareadas que diferiam quanto ao tipo de bobina de radiofreqüência, ambas com sincronização respiratória (caso 15).

(SPIR: com supressão de gordura; SR: sincronização respiratória; BC: bobina de corpo; BS: bobina de sinergia; $\mathrm{IS}_{\mathrm{F}} / \mathrm{R}$ : intensidade de sinal do fígado/ruído de fundo).

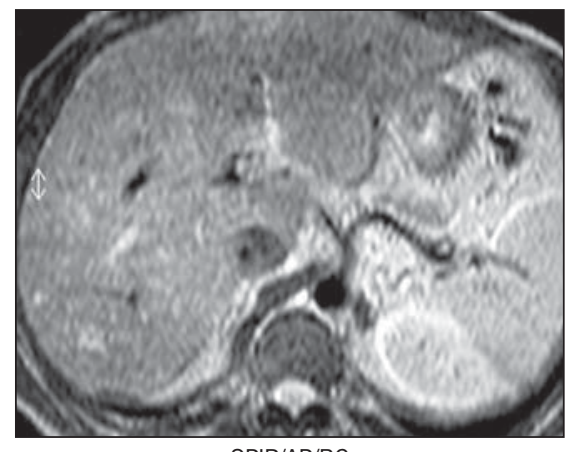

SPIR/AP/BC

Nota $=4$ e IS $/$ R $=15,9$

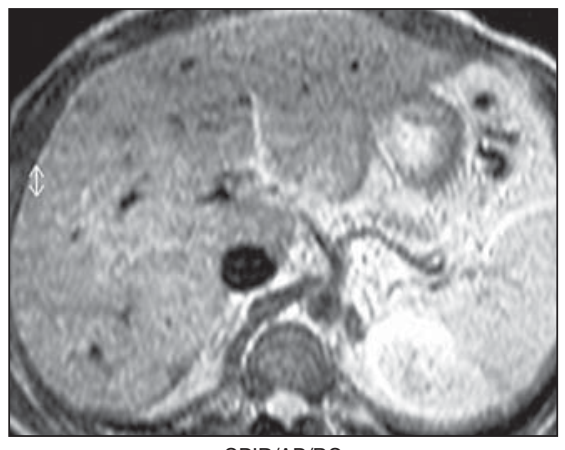

SPIR/AP/BS

Nota $=4$ e IS $/$ R $=4,3$

Figura 11. Exemplificação da avaliação isolada das seqüências pareadas que diferiam quanto ao tipo de bobina de radiofreqüência, ambas em apnéia (caso 19).

(SPIR: com supressão de gordura; AP: apnéia; BC: bobina de corpo; BS: bobina de sinergia; IS $/$ /R: intensidade de sinal do fígado/ruído de fundo).

Contrariamente ao esperado, os resultados apresentados não demonstraram um consenso, com trabalhos favoráveis à sincronização respiratória ${ }^{(20,33)}$, e outros a favor da apnéia ${ }^{(\mathbf{1 8 , 2 9 - 3 2})}$. Como observado em nosso estudo, e em alguns outros na literatura, as imagens obtidas com o sincroni-

zador respiratório apresentaram melhor qualidade e superior relação sinal/ruído que aquelas obtidas durante uma única apnéia $^{(\mathbf{2 0 , 3 3 )}}$. A idade dos pacientes estudados e o seu estado clínico aparentemente não contribuíram para a obtenção de resultados favoráveis ao uso de sincronização respiratória se comparados à adoção de técnica em apnéia. Embora não tenhamos adotado critérios de exclusão referentes ao ritmo respiratório e à capacidade de realizar apnéia, a distribuição por idade neste estudo foi bastante homogênea.

Em nosso estudo, as sequiências realizadas com sincronização respiratória apresentaram resultados significativamente superiores, tanto pela análise qualitativa quanto pela quantitativa, quando comparadas àquelas realizadas em apnéia, em concordância com alguns trabalhos da literatura $^{(\mathbf{2 0 , 3 3 )}}$. Tang et al. $^{(\mathbf{2 0})}$ justificaram seus resultados favoráveis às imagens com sincronização respiratória, devido à maior ocorrência de artefatos de movimento fisiológico nas imagens realizadas em apnéia, pelo seu reduzido tempo de aquisição, com conseqüente relaxamento incompleto dos prótons dos tecidos avaliados. Low et al. ${ }^{(33)}$ explicaram os seus achados pelo ajuste do TR com a freqüência respiratória dos pacientes nas imagens realizadas com sincronização respiratória, e pela adequada calibração do sincronizador respiratório de seu equipamento, fornecendo imagens de elevada qualidade diagnóstica. Em nosso estudo notamos que, independentemente da idade ou da capacidade respiratória dos pacientes, a sincronização respiratória apresentou resultados qualitativos significativamente superiores às imagens obtidas com controle da apnéia.

Entre os trabalhos que demonstraram superioridade qualitativa das imagens obtidas em apnéia, em comparação àquelas com sincronização respiratória, os dois estudos realizados, em 1996, por Soyer et $a l .{ }^{(\mathbf{1 8 , 1 9 )}}$ apresentam algumas limitações relacionadas à metodologia adotada, pois utilizaram tempos de eco e de repetição diferentes nas sequiências obtidas em apnéia e com sincronização respiratória ${ }^{(\mathbf{2 8 , 2 9 )}}$. Em nosso trabalho, em contrapartida, a utilização de parâmetros técnicos divergentes não representa uma limitação metodológica, pois utilizamos TE e TR praticamente iguais em todas as sequiências. No estudo realizado por Kanematsu et al. ${ }^{(\mathbf{3 0})}$, a superioridade da sequiência TSE, em apnéia, foi justificada pela elevada capacidade dos pacientes examinados em manter a apnéia. Os autores não detalham as características clínicas da população estudada 
e a sua capacidade respiratória. Por outro lado, explicam que a degradação das imagens com sincronização respiratória poderia estar relacionada à não utilização de uma técnica de supressão do tecido adiposo; todavia, a supressão de gordura também não foi usada nas sequiências em apnéia correspondentes, não sendo aparentemente uma justificativa pertinente para o insucesso das sequiências com sincronização respiratória. No trabalho de Kim et $a l .{ }^{(31)}$, os autores defendem a redução significativa dos artefatos de respiração e conseqüente melhor qualidade das imagens obtidas em apnéia, pela utilização de bobina de sinergia em seu estudo, diferentemente de todos os outros trabalhos anteriormente citados. O uso de uma bobina de radiofreqüência em contato com a parede abdominal seria o responsável pelo aumento da relação sinal/ruído das imagens obtidas em apnéia, que são intrinsecamente caracterizadas por uma baixa relação sinal/ ruído, devido ao reduzido tempo de aquisição deste tipo de sequiência.

Entre os estudos previamente assinalados, quatro deles também realizaram a análise quantitativa das imagens, com mensuração das intensidades de sinal do fígado e do ruído de fundo, e posterior cálculo da relação entre elas ${ }^{(\mathbf{1 8 , 2 9 , 3 2 , 3 3 )}}$. Apesar dos resultados diferentes observados na análise qualitativa, três destes ${ }^{(\mathbf{1 8 , 2 9 , 3 2 )}}$ foram unânimes quanto à superioridade quantitativa das seqüências realizadas com sincronização respiratória, semelhante ao demonstrado em nosso estudo. É interessante observar que, à semelhança do observado em nosso trabalho, nem sempre existem resultados coincidentes para análises quantitativas e qualitativas, como particularmente demonstrado nas pesquisas de Soyer et al. ${ }^{(\mathbf{1 8 , 3 2})}$. Somente no trabalho realizado por Kim et al. ${ }^{(31)}$ os autores constataram a superioridade qualitativa e quantitativa da técnica em apnéia; porém, como já referido, seus resultados podem ter sido influenciados pela utilização de bobina de sinergia nos exames realizados em apnéia.

O segundo ponto de divergência na literatura, avaliado em nosso trabalho, foi a utilização de uma técnica de supressão de gordura em associação às seqüências TSE ponderadas em T2. As vantagens da utilização concomitante da supressão de gor- dura, observadas em alguns trabalhos, seriam a melhora do poder diagnóstico das imagens obtidas pela supressão da gordura hiperintensa e brilhante do tecido celular subcutâneo e intra-abdominal, o aumento da conspicuidade de detecção de lesões parenquimatosas, e a redução de artefatosfantasma e de "chemical-shift"(11). Estas vantagens deveriam ser ainda mais evidentes nas sequiências TSE, que são caracterizadas por maior intensidade de sinal da gordura, quando comparadas às tradicionais seqüências "spin-echo"(11,31).

Não observamos, em nosso trabalho, diferença estatisticamente significante tanto entre a avaliação qualitativa quanto pela avaliação quantitativa das imagens obtidas com e sem supressão de gordura. Na literatura, a maior controvérsia aparece nos resultados da análise qualitativa, alguns sendo favoráveis à utilização sistemática de supressão de gordura ${ }^{(\mathbf{1 5 , 1 8})}$, enquanto outros

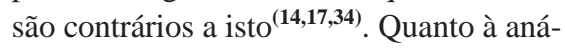
lise quantitativa, a maioria dos trabalhos demonstra relação sinal/ruído superior nas imagens com supressão de gordura ${ }^{(\mathbf{1 4}-18)}$. Como a não utilização da técnica de supressão de gordura também apresenta vantagens, sendo importante para a melhor delimitação anatômica dos contornos dos órgãos, particularmente do fígado, e para o auxílio na caracterização de lesões com conteúdo lipídico, acreditamos que a decisão sobre o uso ou não de supressão de gordura possa ser opcional, pois não haverá prejuízo definitivo na qualidade das imagens, assim como na capacidade diagnóstica destas. Considerando que, para identificar e caracterizar lesões hepáticas focais, são necessárias duas sequiências ponderadas em T2, uma com TE mais curto $(70-90 \mathrm{~ms})$ e outra com TE mais longo $(140-160 \mathrm{~ms})^{(\mathbf{5 , 3 5}-38)}$, sugerimos que uma seja realizada com e a outra sem a técnica de supressão de gordura.

Em nosso estudo, as seqüências com bobina de sinergia apresentaram qualidade de imagem semelhante às obtidas com bobina de corpo, embora estas últimas tenham apresentado uma melhor relação sinal/ruído em comparação com as primeiras, quando realizadas em apnéia. Somente três trabalhos na literatura ${ }^{(\mathbf{2 3 , 2 4 , 3 4 )}}$ compararam o uso dessas duas bobinas em seqüências TSE ponderadas em T2, demons- trando uma superioridade das seqüências com bobina de sinergia, através da análise quantitativa das imagens, diferentemente dos nossos resultados. Somente o estudo realizado por Campeau et al. ${ }^{\text {(24) }}$ também compara estes dois tipos de bobina de radiofreqüência em termos de qualidade de imagem. As imagens com bobina de sinergia permitiram a detecção de um maior número de estruturas anatômicas intra-abdominais, porém não encontraram diferença estatisticamente significante em relação à ocorrência de artefatos de respiração e de pulsação vascular.

Avaliando a divergência existente entre os nossos resultados e os apresentados em outros estudos, quanto à superioridade de um determinado tipo de bobina, podemos tecer algumas considerações. Primeiramente, diversos autores, ao comparar os dois tipos de bobina (quadratura e sinergia) adotaram diferentes tipos de seqüências de pulso, o que provavelmente interfere na análise dos resultados obtidos. Em seu estudo, Campeau et al. ${ }^{(\mathbf{2 4})}$, por exemplo, compararam quatro seqüências: GRE ponderada em T1, SE convencional ponderada em T2, FSE em apnéia e eco planar; Schwartz et al. ${ }^{(\mathbf{2 3})}$ utilizaram as sequiências FSE ponderada em T2 e "fast multiplanar inversion recovery"; e Kim et al. ${ }^{(34)}$, por sua vez, as seqüências TSE em apnéia e HASTE ponderadas em T2. Ao contrário, procuramos utilizar não somente a mesma sequiência (TSE), mas até mesmo parâmetros técnicos semelhantes. Dessa forma, a utilização de seqüências perfeitamente pareadas, tendo como única variável o tipo de bobina utilizada, valoriza e valida os resultados obtidos em nosso trabalho.

A introdução das bobinas de sinergia na prática clínica foi justificada pelos bons resultados encontrados em estudos experimentais. A elevada relação sinal/ruído destas bobinas é fruto do seu contato com a superfície corporal em estudo e da existência de múltiplos elementos no interior destas bobinas, que captam de forma combinada e aditiva o sinal emitido pelos tecidos. Estas características permitem obter imagens com elevada definição diagnóstica. Apesar dos argumentos irrefutáveis, não obtivemos, em nosso trabalho, resultados que confirmem a superioridade da bobina de sinergia em estudos hepáticos, 
utilizando o tipo de equipamento adotado nesta pesquisa. Podemos aventar algumas hipóteses que justifiquem tais achados, entre elas o fato de a bobina de sinergia utilizada ser uma das primeiras a serem instaladas neste tipo de equipamento, e, possivelmente, merecer ajuste e calibração mais detalhados. Outro fato a se considerar é a elevada e reconhecida qualidade da bobina de quadratura que equipa o aparelho de RM fabricado pela Philips.

Nos três estudos previamente citados, que utilizaram bobina de sinergia, a técnica empregada para controle dos artefatos de respiração nas sequiências TSE ponderadas em T2 foi a apnéia, provavelmente com o objetivo de adquirir imagens em curto tempo e com relação sinal/ruído compensada pelo uso de bobina de superfície. Vale ressaltar, todavia, a existência de um trabalho na literatura ${ }^{(39)}$, que, utilizando somente bobina de sinergia, comparou a sincronização respiratória e a apnéia e demonstrou resultados superiores da sincronização respiratória, tanto pela análise qualitativa quanto pela quantitativa. Em nosso trabalho, a comparação isolada das seqüências com bobina de sinergia, ambas com supressão de gordura e diferindo quanto à técnica de controle respiratório, demonstrou a superioridade da seqüência com sincronização respiratória.

Quando as seis sequiências realizadas neste estudo foram classificadas, de acordo com a análise qualitativa das imagens obtidas, as melhores foram a SPIR/SR/BC e a s/SPIR/SR/BC. Estas seqüências apresentam, em comum, o uso de sincronização respiratória e bobina de quadratura, porém, diferem quanto à realização ou não de supressão de gordura. Dessa forma, e como já aventado previamente, diante dos resultados obtidos com a técnica de exame aqui apresentada, sugerimos o uso de bobina de corpo e de sincronização respiratória, independentemente da utilização ou não de supressão de gordura, com a finalidade de se obterem imagens hepáticas pela seqüência TSE ponderada em T2, com elevada qualidade diagnóstica.

Ressalta-se que, entre as seis sequiências realizadas neste trabalho, as que ocuparam as três primeiras colocações, através da análise qualitativa, foram aquelas que utilizaram a sincronização respiratória, de- monstrando mais uma vez ser este o parâmetro técnico que apresentou os resultados mais significativos sob o ponto de vista estatístico. Mesmo com o uso de bobina de sinergia, as imagens com sincronização respiratória foram melhores. Este dado também é divergente dos encontrados em alguns trabalhos na literatura, que sugeriram a utilização da apnéia combinada à bobina de sinergia ${ }^{(\mathbf{2 4 , 3 4 )}}$. Estes estudos procuraram justificar os seus resultados, através de hipótese em que a reduzida relação sinal/ruído das imagens obtidas no curso de uma única apnéia poderia ser compensada com o uso da bobina de superfície. Não acreditamos que esta justificativa seja relevante, pois o uso de bobina de sinergia aliado à sincronização respiratória, que, segundo os próprios autores destes trabalhos, apresenta uma relação sinal/ruído superior à apnéia, também beneficiaria as imagens obtidas com bobina de sinergia e sincronização respiratória, como foi observado em nossos resultados.

Quando as seqüências realizadas em nosso estudo foram graduadas pela análise quantitativa, através do cálculo da relação sinal/ruído das imagens, as melhores também foram aquelas obtidas com sincronização respiratória (SPIR/SR/BC e s/SPIR/ SR/BC). Nesta avaliação, todavia, a seqüência SPIR/AP/BC não apresentou diferença estatística significante em relação às duas primeiras. Vale ressaltar, todavia, que esta mesma sequiência foi classificada em último lugar pela análise qualitativa das imagens. Embora este achado tenha apresentado significância estatística, acreditamos que a utilização de uma seqüência de pulso, com o intuito de obter imagens hepáticas com poder diagnóstico, deva apresentar também boa delimitação dos contornos do fígado e adequada definição das estruturas anatômicas, bem como uma baixa ocorrência de artefatos de respiração, elementos que foram avaliados em nossa análise qualitativa das imagens.

Neste trabalho, as seqüências com sincronização respiratória e com bobina de corpo, utilizando ou não supressão de gordura, foram as melhores dentre as duas análises realizadas. A seqüência com supressão de gordura, em apnéia e com bobina de corpo, embora tenha tido um bom desempenho pela avaliação quantitativa, ficou em último lugar na qualitativa, não sendo desta forma considerada uma opção adequada para a obtenção de imagens de RM ponderadas em $\mathrm{T} 2$, fundamentais para o diagnóstico das doenças do fígado.

\section{REFERÊNCIAS}

1. Curati WL, Bydder GM. Magnetic resonance imaging: present position and future prospects. Eur J Cancer 1996;32A:589-92.

2. Doyle FH, Pennock JM, Banks LM, et al. Nuclear magnetic resonance imaging of the liver: initial experience. AJR 1982;138:193-200.

3. Mahfouz AE, Hamm B, Taupitz M. Hepatic magnetic resonance imaging: new techniques and contrast agents. Endoscopy 1997;29:504-14.

4. Ichikawa T, Araki T. Fast magnetic resonance imaging of liver. Eur J Radiol 1999;29:186-210.

5. Weissleder R, Stark DD. Magnetic resonance imaging of liver tumors. Semin Ultrasound CT MR 1989;10:63-77.

6. Schima W, Saini S, Echeverri JA, Hahn PF, Harisinghani M, Mueller PR. Focal liver lesions: characterization with conventional spin-echo versus fast spin-echo T2-weighted MR imaging. Radiology 1997;202:389-93.

7. Riederer SJ. Recent technical advances in MR imaging of the abdomen. J Magn Reson Imaging 1996;6:822-32.

8. Ferrucci JT. Advances in abdominal MR imaging. RadioGraphics 1998;18:1569-86.

9. Princípios básicos de imagens por RM. Manual didático do equipamento de RM do fabricante Philips, 2000

10. Mirowitz SA. Rapid abdominal MR imaging. Magn Reson Imaging Clin N Am 1995;3:1-12.

11. Kanematsu M, Hoshi H, Itoh K, et al. Focal hepatic lesion detection: comparison of four fat-suppressed T2-weighted MR imaging pulse sequences. Radiology 1999;211:363-71.

12. Mitchell DG, Vinitski S, Burk DL Jr, Levy DW, Rifkin MD. Motion artifact reduction in MR imaging of the abdomen: gradient moment nulling versus respiratory-sorted phase encoding. Radiology 1988;169:155-60.

13. Semelka RC, Chew W, Hricak H, Tomei E, Higgins CB. Fat-saturation MR imaging of the upper abdomen. AJR 1990;155:1111-6.

14. Schwartz LH, Seltzer SE, Tempany CM, et al. Prospective comparison of T2-weighted fast spin-echo, with and without fat suppression, and conventional spin-echo pulse sequences in the upper abdomen. Radiology 1993;189:411-6.

15. Lu DS, Saini S, Hahn PF, et al. T2-weighted MR imaging of the upper part of the abdomen: should fat suppression be used routinely? AJR 1994;162: 1095-100.

16. Kreft B, Layer G, Kuhl C, Sommer T, Gieseke J, Schild H. Turbo-Spin-Echo-Sequenzen mit selektiver Fettunterdruckung (SPIR) in der MRT von fokalen Leberlasionen bei 0.5 Tesla. Rofo Fortschr Geb Rontgenstr Neuen Bildgeb Verfahr 1995; 163 : 411-6.

17. Yu JS, Kim KW, Kim YH, Jeong EK, Chien D. Comparison of multishot turbo spin echo and HASTE sequences for T2-weighted MRI of liver lesions. J Magn Reson Imaging 1998;8:1079-84.

18. Soyer P, Le Normand S, de Givry SC, Gueye C, Somveille E, Scherrer A. T2-weighted spin-echo 
MR imaging of the liver: breath-hold fast spin-echo versus non-breath-hold fast spin-echo images with and without fat suppression. AJR 1996;166:5937.

19. Soyer P, Gouhiri M, Rondeau Y, Spelle L, Mosnier $H$, Scherrer A. Non-breath-hold fast spin-echo versus breath-hold fast spin-echo and spoiled gradientrecalled echo MR imaging in the detection of hepatic tumors: correlation with surgical findings. AJR 1997;168:1199-204.

20. Tang Y, Yamashita Y, Namimoto T, Abe Y, Takahashi M. Liver T2-weighted MR imaging: comparison of fast and conventional half-Fourier singleshot turbo spin-echo, breath-hold turbo spin-echo, and respiratory-triggered turbo spin-echo sequences. Radiology 1997;203:766-72.

21. Zee CS, Segall HD, Terk MR, et al. SPIR MRI in spinal diseases. J Comput Assist Tomogr 1992;16: 356-60.

22. Yu KK, Hricak H, Subak LL, Zaloudek CJ, Powell CB. Preoperative staging of cervical carcinoma: phased array coil fast spin-echo versus body coil spin-echo T2-weighted MR imaging. AJR 1998; 171:707-11.

23. Schwartz LH, Panicek DM, Thomson E, et al. Comparison of phased-array and body coils for MR imaging of liver. Clin Radiol 1997;52:745-9.

24. Campeau NG, Johnson CD, Felmlee JP, et al. MR imaging of the abdomen with a phased-array multicoil: prospective clinical evaluation. Radiology
1995;195:769-76.

25. Goldsmith NA, Woodburne, RT. The surgical anatomy pertaining to liver resection. Surg Gynecol Obstet 1957;105:310-8.

26. Bismuth H. Surgical anatomy and anatomical surgery of the liver. World J Surg 1982;6:3-9.

27. Landis JR, Koch GG. The measurement of observer agreement for categorical data. Biometrics 1977; 33:159-74.

28. Papanikolaou N, Moulopoulos LA, Gouliamos A, Ispanopoulou S, Vlahos L. Comparison of dual spin echo echo planar imaging (SE-EPI), turbo spin echo with fat suppression and conventional dual spin echo sequences for T2-weighted MR imaging of focal liver lesions. Magn Reson Imaging 2000;18: 715-9.

29. Saini S, Nelson RC. Technique for MR imaging of the liver. Radiology 1995;197:575-7.

30. Kanematsu M, Hoshi H, Murakami T, et al. Focal hepatic lesion detection: comparison of four T2 weighted MR imaging pulse sequences. Radiology 1998;206:167-75.

31. Kim TK, Wang WC, Han JK, Cho SG, Choi BI. T2weighted MR imaging for hepatic hemangiomas: comparison of breath-hold and non-breath-hold turbo spin-echo pulse sequences with phased-array multicoil. Abdom Imaging 1998;23:422-6.

32. Soyer P, de Givry SC, Gueye C, Le Normand S, Somveille E, Scherrer A. Detection of focal hepatic lesions with MR imaging: prospective comparison of T2-weighted fast spin-echo with and without fat suppression, T2-weighted breath-hold fast spinecho, and gadolinium chelate-enhanced 3D gradient-recalled imaging. AJR 1996;166:1115-21.

33. Low RN, Alzate GD, Shimakawa A. Motion suppression in MR imaging of the liver: comparison of respiratory-triggered and nontriggered fast spinecho sequences. AJR 1997;168:225-31.

34. Kim TK, Lee HJ, Jang HJ, Kim AY, Han JK, Choi BI. T2-weighted breath-hold MRI of the liver at 1.0 T: comparison of turbo spin-echo and HASTE sequences with and without fat suppression. J Magn Reson Imaging 1998;8:1213-8.

35. Laing AD, Gibson RN. MRI of the liver. J Magn Reson Imaging 1998;8:337-45

36. Foley WD, Kneeland JB, Cates JD, et al. Contrast optimization for the detection of focal hepatic lesions by MR imaging at $1.5 \mathrm{~T}$. AJR 1987;149: 1155-60.

37. Robinson PJ. The characterization of liver tumors by MRI. Clin Radiol 1996;51:749-61.

38. Ito K, Mitchell DG, Outwater EK, Szklaruk J, Sadek AG. Hepatic lesions: discrimination of nonsolid, benign lesions from solid, malignant lesions with heavily T2-weighted fast spin-echo MR imaging. Radiology 1997;204:729-37.

39. Namimoto T, Yamashita Y, Mitsuzaki K, Takahashi $M$. The value of respiratory-triggered T2-weighted turbo spin-echo imaging of the liver using a phased array coil. J Magn Reson Imaging 1998;8:655-62. 